Kragujevac Journal of Mathematics

Volume 44(3) (2020), Pages 379-392.

\title{
AN APPROXIMATE APPROACH FOR SYSTEMS OF FRACTIONAL INTEGRO-DIFFERENTIAL EQUATIONS BASED ON TAYLOR EXPANSION
}

\author{
M. DIDGAR ${ }^{1,2}$, A. R. VAHIDI ${ }^{3 *}$, AND J. BIAZAR $^{4}$
}

\begin{abstract}
The main purpose of this work is to present an efficient approximate approach for solving linear systems of fractional integro-differential equations based on a new application of Taylor expansion. Using the $m$ th-order Taylor polynomial for unknown functions and employing integration method the given system of fractional integro-differential equations will be converted into a system of linear equations with respect to unknown functions and their derivatives. The solutions of this system yield the approximate solutions of fractional integro-differential equations system. The Riemann-Liouville fractional derivative is applied in calculations. An error analysis is discussed as well. The accuracy and the efficiency of the suggested method is illustrated by considering five numerical examples.
\end{abstract}

\section{INTRODUCTION}

During the past decades, fractional calculus and fractional differential equations have found various applications in sciences and engineering, such as electrical networks, rheology, acoustics, electroanalytical chemistry, neuron modeling, viscoelasticity, material sciences, fluid flow, diffusive transport akin to diffusion, probability, electromagnetic theory, and so on (see [7,13,18,24, 26]).

Since most of FDEs do not have exact solutions, approximate and numerical techniques have received considerable attention to solve fractional differential equations.

Key words and phrases. Fractional differential equation (FDE), systems of fractional integrodifferential equations (SFIDE), Riemann-Liouville fractional derivative, Taylor expansion, error analysis.

2010 Mathematics Subject Classification. Primary: 65R20. Secondary: 34A08.

DOI 10.46793/KgJMat2003.379D

Received: March 22, 2018.

Accepted: May 22, 2018. 
So far, several analytical and numerical methods have been proposed to solve fractional differential equations which the interested reader can refer to $[1-5,10-12,16,19$ $23,25,27-30,34]$ and the references therein.

In this paper, we investigate the approximate solutions of linear fractional integrodifferential equations systems based on a new application of Taylor expansion (see $[6,8-10,14,15,17,31-33])$. By expanding unknown functions as an $m$ th-order Taylor polynomial and employing integration method, we can convert the given system of fractional integro-differential equations into a system of linear equations with respect to unknown functions and their derivatives. Approximate solutions can be obtained by solving the resulting system of equations according to a standard rule. The results of the obtained approximations of the suggested method are then compared with the referenced methods for several examples. In the present investigation, the main property of this approximate method besides simplicity and reliability is that an $m$ thorder approximation is equal to exact solution if the exact solution is a polynomial of degree at most $m$. The present work may be viewed as an extension of the results obtained in [10].

The remainder of this paper is organized as follows. In Section 2, some definitions of fractional calculus are recalled. In Section 3, we describe the proposed method. In Section 4, we give an error analysis. In Section 5, we investigate some examples, which demonstrate the effectiveness of our approach. In Section 6, our findings are concluded.

\section{Preliminaries and Basic Definitions}

Let's describe some basic concepts, and properties of the fractional calculus, which will be used later.

Definition 2.1. A real function $\phi(x), x>0$, is said to be in the space $C_{\mu}, \mu \in \mathbb{R}$ if there exists a real number $p(>\mu)$, such that $\phi(x)=x^{p} \phi_{1}(x)$, where $\phi_{1}(x) \in C[0, \infty)$, and it is said to be in the space $C_{\mu}^{n}$ if and only if $\phi^{(n)} \in C_{\mu}, n \in \mathbb{N}$.

Definition 2.2. The Riemann-Liouville fractional integral operator of order $\alpha \geq 0$, of a function $\phi \in C_{\mu}, \mu \geq-1$, is considered as follows

$$
\begin{aligned}
J^{\alpha} \phi(x) & =\frac{1}{\Gamma(\alpha)} \int_{0}^{x}(x-t)^{\alpha-1} \phi(t) d t, \quad \alpha>0, x>0, \\
J^{0} \phi(x) & =\phi(x) .
\end{aligned}
$$

Definition 2.3. The Caputo fractional derivative of $\phi(x)$ is considered as follows

$$
D_{*}^{\alpha} \phi(x)=J^{n-\alpha}\left(\frac{d^{n}}{d x^{n}} \phi(x)\right)=\frac{1}{\Gamma(n-\alpha)} \int_{0}^{x}(x-t)^{n-\alpha-1} \phi^{(n)}(t) d t,
$$

for $n-1<\alpha \leq n, n \in \mathbb{N}, x>0, \phi \in C_{-1}^{n}$. 
Definition 2.4. The Riemann-Liouville fractional derivative of $\phi(x)$ is considered as follows

for $n-1<\alpha \leq n, n \in \mathbb{N}, x>0, \phi \in C_{-1}^{n}$.

$$
D^{\alpha} \phi(x)=\frac{d^{n}}{d x^{n}}\left(J^{n-\alpha} \phi(x)\right)
$$

\section{Description of the Method}

Consider the following system of linear fractional integro-differential equations

$$
\begin{aligned}
& D^{\alpha_{i}} \psi_{i}(x)+\lambda_{1} \int_{0}^{1} \sum_{j=1}^{\nu} K_{1_{i j}}(x, t) \psi_{j}(t) d t+\lambda_{2} \int_{0}^{x} \sum_{j=1}^{\nu} K_{2_{i j}}(x, t) \psi_{j}(t) d t \\
= & f_{i}(x), \quad i=1, \ldots, \nu,
\end{aligned}
$$

with initial conditions

$$
\psi_{i}^{(\kappa)}(0)=0, \quad \kappa=0,1, \ldots, n-1, n-1<\alpha_{i} \leq n, n \in \mathbb{N},
$$

where $D^{\alpha_{i}} \psi_{i}(x)$ indicates Riemann-Liouville fractional derivative of order $\alpha_{i}$, and $\lambda_{1}$, $\lambda_{2}$ are constants, $K_{1_{i j}}(x, t), K_{2_{i j}}(x, t), f_{i}(x)$ are given known functions which satisfy certain conditions so that system (3.2) has a unique solution, and $\psi_{i}(x)$ are unknown functions.

According to definition (2.4), system of fractional integro-differential equation (3.1) can be rewritten as

$$
\frac{d^{n}}{d x^{n}}\left(J^{n-\alpha_{i}} \psi_{i}(x)\right)+\lambda_{1} \int_{0}^{1} \sum_{j=1}^{\nu} K_{1_{i j}}(x, t) \psi_{j}(t) d t+\lambda_{2} \int_{0}^{x} \sum_{j=1}^{\nu} K_{2_{i j}}(x, t) \psi_{j}(t) d t=f_{i}(x)
$$

or equivalently by using definition (2.2), we have

$$
\begin{aligned}
& \frac{d^{n}}{d x^{n}}\left(\frac{1}{\Gamma\left(n-\alpha_{i}\right)} \int_{0}^{x}(x-t)^{n-\alpha_{i}-1} \psi_{i}(t) d t\right)+\lambda_{1} \int_{0}^{1} \sum_{j=1}^{\nu} K_{1_{i j}}(x, t) \psi_{j}(t) d t \\
& +\lambda_{2} \int_{0}^{x} \sum_{j=1}^{\nu} K_{2_{i j}}(x, t) \psi_{j}(t) d t=f_{i}(x) .
\end{aligned}
$$

In the following, by integrating both hand side of (3.3), $n$ times with respect to $x$ from 0 to $s$ and with the help of changing the order of the integrations, we obtain

$$
\begin{aligned}
& \frac{1}{\Gamma\left(n-\alpha_{i}\right)} \int_{0}^{x}(x-t)^{n-\alpha_{i}-1} \psi_{i}(t) d t+\lambda_{1} \sum_{j=1}^{\nu} \int_{0}^{1} \int_{0}^{x} \frac{(x-s)^{l-1}}{(l-1) !} K_{1_{i j}}(s, t) \psi_{j}(t) d s d t \\
& +\lambda_{2} \sum_{j=1}^{\nu} \int_{0}^{x} \int_{t}^{x} \frac{(x-s)^{l-1}}{(l-1) !} K_{2_{i j}}(s, t) \psi_{j}(t) d s d t=F_{i}(x), \quad l=1, \ldots, n
\end{aligned}
$$

where

$$
F_{i}(x)=\int_{0}^{x} \frac{(x-t)^{l-1}}{(l-1) !} f_{i}(t) d t, \quad i=1, \ldots, \nu,
$$

in which the variable $s$ has been replaced by $x$, for simplicity. Hence we transformed the system of fractional integro-differential equations (3.1) into a system of mixed 
Volterra-Fredholm integral equations. To approximately solve the resulting system, we reduce Eq. (3.4) into a system of linear equations with respect to unknown functions and their derivatives. Toward this goal, the method assumes that the desired solutions $\psi_{j}(t)$ to be $m+1$ times continuously differentiable on the interval $I$, in other words $\psi_{j} \in C^{m+1}(I)$. Therefore, for $\psi_{j} \in C^{m+1}(I), \psi_{j}(t)$ can be expressed in terms of the $m$ th-order Taylor series at an arbitrary point $x \in I$ as

$$
\psi_{j}(t)=\psi_{j}(x)+\psi_{j}^{\prime}(x)(t-x)+\cdots+\frac{1}{m !} \psi_{j}^{(m)}(x)(t-x)^{m}+E_{j, m}(t, x),
$$

where $E_{j, m}(t, x)$ indicates the Lagrange error bound

$$
E_{j, m}(t, x)=\frac{\psi_{j}^{(m+1)}\left(\xi_{j}\right)}{(m+1) !}(t-x)^{m+1},
$$

for some point $\xi_{j}$ between $x$ and $t$. Generally, the Lagrange error bound $E_{j, m}(t, x)$ becomes sufficiently small as $m$ gets great enough. Especially, if the solutions $\psi_{j}(t)$ are polynomials of degree up to $m$, then the last Lagrange error bound becomes zero, namely, the obtained approximate solutions of system (3.1) yield the true solutions. With due attention to aforementioned assumption, by omitting the last Lagrange error bound, we consider the truncated Taylor expansion $\psi_{j}(t)$ as

$$
\psi_{j}(t) \approx \sum_{k=0}^{m} \psi_{j}^{(k)}(x) \frac{(t-x)^{k}}{k !} .
$$

Inserting the approximate relation (3.5), for unknown functions $\psi_{j}(t)$, into (3.4) we obtain

$$
\begin{aligned}
& \sum_{k=0}^{m} \frac{(-1)^{k}}{k !} \psi_{j}^{(k)}(x) \int_{0}^{x} \frac{(x-t)^{k+n-\alpha_{i}-1}}{\Gamma\left(n-\alpha_{i}\right)} d t \\
& +\lambda_{1} \sum_{j=1}^{\nu} \sum_{k=0}^{m} \frac{\psi_{j}^{(k)}(x)}{k !} \int_{0}^{1} \int_{0}^{x} \frac{(x-s)^{l-1}}{(l-1) !}(t-x)^{k} K_{1_{i j}}(s, t) d s d t \\
& +\lambda_{2} \sum_{j=1}^{\nu} \sum_{k=0}^{m} \frac{\psi_{j}^{(k)}(x)}{k !} \int_{0}^{x} \int_{t}^{x} \frac{(x-s)^{l-1}}{(l-1) !}(t-x)^{k} K_{2_{i j}}(s, t) d s d t \\
& =F_{i}(x), \quad i=1, \ldots, \nu .
\end{aligned}
$$

In fact, system (3.1) was converted into a linear system of ordinary differential equations with respect to $\psi_{j}(x)$ and its derivatives up to order $m$. In other word, we have obtained $\nu$ linear equations in (3.6) with respect to $\nu \times(m+1)$ unknown functions $\psi_{j}^{(k)}$, for $k=0, \ldots, m, j=1, \ldots, \nu$. In the following, we want to determine $\psi_{j}^{(k)}$ by solving a system of linear equations. In order to achieve this goal, other $\nu \times m$ independent linear equations with respect to $\psi_{j}(x), \ldots, \psi_{j}^{(m)}(x)$ are needed, which can be achieved by integrating both sides of Eq.(3.4) $m$ times with respect to $x$. Thus, 
we have

$$
\begin{aligned}
& \int_{0}^{x} \frac{(x-t)^{\gamma+n-\alpha_{i}-1}}{\Gamma\left(\gamma+n-\alpha_{i}\right)} \psi_{i}(t) d t+\lambda_{1} \sum_{j=1}^{\nu} \int_{0}^{1} \int_{0}^{x} \frac{(x-s)^{\gamma+l-1}}{(\gamma+l-1) !} K_{1_{i j}}(s, t) \psi_{j}(t) d s d t \\
& +\lambda_{2} \sum_{j=1}^{\nu} \int_{0}^{x} \int_{t}^{x} \frac{(x-s)^{\gamma+l-1}}{(\gamma+l-1) !} K_{2_{i j}}(s, t) \psi_{j}(t) d s d t=F_{i}^{(\gamma)}(x), \quad \gamma=1, \ldots, m,
\end{aligned}
$$

where

$$
F_{i}^{(\gamma)}(x)=\int_{0}^{x} \frac{(x-t)^{\gamma-1}}{(\gamma-1) !} F_{i}(t) d t, \quad i=1, \ldots, \nu, \gamma=1, \ldots, m .
$$

We apply the Taylor expansion again and substituting (3.5) for $\psi_{j}(t)$ into $\mathrm{E}(3.7)$ leads to

$$
\begin{aligned}
& \sum_{k=0}^{m} \frac{(-1)^{k}}{k !} \psi_{j}^{(k)}(x) \int_{0}^{x} \frac{(x-t)^{k+\gamma+n-\alpha_{i}-1}}{\Gamma\left(\gamma+n-\alpha_{i}\right)} d t \\
& +\lambda_{1} \sum_{j=1}^{\nu} \sum_{k=0}^{m} \frac{\psi_{j}^{(k)}(x)}{k !} \int_{0}^{1} \int_{0}^{x} \frac{(x-s)^{\gamma+l-1}}{(\gamma+l-1) !}(t-x)^{k} K_{1_{i j}}(s, t) d s d t+ \\
& \lambda_{2} \sum_{j=1}^{\nu} \sum_{k=0}^{m} \frac{\psi_{j}^{(k)}(x)}{k !} \int_{0}^{x} \int_{t}^{x} \frac{(x-s)^{\gamma+l-1}}{(\gamma+l-1) !}(t-x)^{k} K_{2_{i j}}(s, t) d s d t \\
= & F_{i}^{(\gamma)}(x), \quad \gamma=1, \ldots, m .
\end{aligned}
$$

In this way, (3.4) and (3.8) construct a system of linear equations with resect to unknown functions $\psi_{j}(x)$ and its derivatives up to order $m$. The obtained system is indicated as follows

$$
\mathbf{Q}(x) \Psi(x)=F(x)
$$

where

$$
\begin{gathered}
\left(3.9 \mathbf{Q}(x)=\left[\begin{array}{ccccccccccc}
q_{10}^{10}(x) & \cdots & q_{\nu 0}^{10}(x) & \cdots & q_{1 k}^{10}(x) & \cdots & q_{\nu k}^{10}(x) & \cdots & q_{1 m}^{10}(x) & \cdots & q_{\nu m}^{10}(x) \\
\vdots & \ddots & \vdots & \ddots & \vdots & \ddots & \vdots & \ddots & \vdots & \ddots & \vdots \\
q_{10}^{\nu 0}(x) & \cdots & q_{\nu 0}^{\nu 0}(x) & \cdots & q_{1 k}^{\nu 0}(x) & \cdots & q_{\nu k}^{\nu 0}(x) & \cdots & q_{1 m}^{\nu 0}(x) & \cdots & q_{\nu m}^{\nu 0}(x) \\
\vdots & \ddots & \vdots & \ddots & \vdots & \ddots & \vdots & \ddots & \vdots & \ddots & \vdots \\
q_{10}^{1 \gamma}(x) & \cdots & q_{\nu 0}^{1 \gamma}(x) & \cdots & q_{1 k}^{1 \gamma}(x) & \cdots & q_{\nu k}^{1 \gamma}(x) & \cdots & q_{1 m}^{1 \gamma}(x) & \cdots & q_{\nu m}^{1 \gamma}(x) \\
\vdots & \ddots & \vdots & \ddots & \vdots & \ddots & \vdots & \ddots & \vdots & \ddots & \vdots \\
q_{10}^{\nu \gamma}(x) & \cdots & q_{\nu 0}^{\nu \gamma}(x) & \cdots & q_{1 k}^{\nu \gamma}(x) & \cdots & q_{\nu k}^{\nu \gamma}(x) & \cdots & q_{1 m}^{\nu \gamma}(x) & \cdots & q_{\nu m}^{\nu \gamma}(x) \\
\vdots & \ddots & \vdots & \ddots & \vdots & \ddots & \vdots & \ddots & \vdots & \ddots & \vdots \\
q_{10}^{1 m}(x) & \cdots & q_{\nu 0}^{1 m}(x) & \cdots & q_{1 k}^{1 m}(x) & \cdots & q_{\nu k}^{1 m}(x) & \cdots & q_{1 m}^{1 m}(x) & \cdots & q_{\nu m}^{1 m}(x) \\
\vdots & \ddots & \vdots & \ddots & \vdots & \ddots & \vdots & \ddots & \vdots & \ddots & \vdots \\
q_{10}^{\nu m}(x) & \cdots & q_{\nu 0}^{\nu m}(x) & \cdots & q_{1 k}^{\nu m}(x) & \cdots & q_{\nu k}^{\nu m}(x) & \cdots & q_{1 m}^{\nu m}(x) & \cdots & q_{\nu m}^{\nu m}(x)
\end{array}\right],\right. \\
\mathbf{F}(x)=\left[\begin{array}{c}
F_{1}(x), \ldots, F_{\nu}(x), \ldots, F_{1}^{(\gamma)}(x), \ldots, F_{\nu}^{(\gamma)}(x), \ldots, F_{1}^{(m)}(x), \ldots, F_{\nu}^{(m)}(x)
\end{array}\right]^{T},
\end{gathered}
$$


$\boldsymbol{\Psi}(x)=\left[\psi_{1}(x), \ldots, \psi_{\nu}(x), \ldots, \psi_{1}^{(k)}(x), \ldots, \psi_{\nu}^{(k)}(x), \ldots, \psi_{1}^{(m)}(x), \ldots, \psi_{\nu}^{(m)}(x)\right]^{T}$.

In coefficient matrix (3.9), the first $\nu$ rows refer to coefficients of $\psi_{j}^{(k)}(x)$ in (3.4) for $k=0, \ldots, m, j=1, \ldots, \nu$ and the other rows refer to coefficients of $\psi_{j}^{(k)}(x)$ in $(3.8)$ for $\gamma=1, \ldots, m$. Application of a standard rule to the resulting new system yields an $m$ th-order approximate solution of $(3.1)$ as $\psi_{i m}(x)$. It is to be noted that not only $\psi_{j}(x)$ but also $\psi_{j}^{(k)}(x)$, for $k=1, \ldots, m$, are determined by solving the resulting new system but in point of fact, it is $\psi_{j}(x)$ that we need.

\section{ERror AnALYSIS}

In this section, we expand the error analysis proposed in [9] for derived $m$ th-order approximate solution of fractional integro-differential equations system (3.1). We assume that the exact solutions $\psi_{j}(t)$ are infinitely differentiable on the interval $I$; so $\psi_{j}(t)$ can be expressed as an uniformly convergent Taylor series in $I$ as follows

$$
\psi_{j}(t)=\sum_{k=0}^{\infty} \psi_{j}^{(k)}(x) \frac{(t-x)^{k}}{k !} .
$$

Using the proposed method in the previous section, system of fractional integrodifferential equations (3.1) can be converted into an equivalent system of linear equations with respect to unknown functions $\psi_{i}^{(k)}(x), k=0,1, \ldots$ as

$$
\mathrm{Q} \Psi=\mathbf{F},
$$

where

$$
\mathbf{Q}=\lim _{\nu \longrightarrow \infty} \mathbf{Q}_{\nu \nu}^{\nu \nu}, \quad \Psi=\lim _{\nu \longrightarrow \infty} \Psi_{\nu}, \quad \mathbf{F}=\lim _{\nu \longrightarrow \infty} \mathbf{F}_{\nu},
$$

in which $\mathbf{Q}_{\nu \nu}^{\nu \nu}, \mathbf{\Psi}_{\nu}$, and $\mathbf{F}_{\nu}$, as shown in the previous section, are defined as follows

$$
\mathbf{Q}_{\nu \nu}^{\nu \nu}=\left[q_{i j}^{p q}(x)\right]_{\nu(m+1) \times \nu(m+1)}, \mathbf{\Psi}_{\nu}=\left[\psi_{i}^{(k)}(x)\right]_{\nu(m+1) \times 1}, \mathbf{F}_{\nu}=\left[f_{i}^{(l)}(x)\right]_{\nu(m+1) \times 1} .
$$

Hence, under the solvability conditions for the above system and letting $\mathbf{B}=\mathbf{Q}^{-1}$, the unique solution is represented as

$$
\Psi=\mathrm{BF} .
$$

We rewrite relation (4.1) in an alternative matrix form as

$$
\left[\begin{array}{c}
\boldsymbol{\Psi}_{\nu} \\
\boldsymbol{\Psi}_{\infty}
\end{array}\right]=\left[\begin{array}{cc}
\mathbf{B}_{\nu \nu}^{\nu \nu} & \mathbf{B}_{\nu \infty}^{\nu \infty} \\
\mathbf{B}_{\infty \nu}^{\infty \nu} & \mathbf{B}_{\infty \infty}^{\infty \infty}
\end{array}\right]\left[\begin{array}{c}
\mathbf{F}_{\nu} \\
\mathbf{F}_{\infty}
\end{array}\right]
$$

Accordingly, we can find out that the vector $\boldsymbol{\Psi}_{\nu}$ consists of the first $\nu(m+1)$ elements of the exact solution vector $\boldsymbol{\Psi}$ must satisfy the following relation

$$
\boldsymbol{\Psi}_{\nu}=\mathbf{B}_{\nu \nu}^{\nu \nu} \mathbf{F}_{\nu}+\mathbf{B}_{\nu \infty}^{\nu \infty} \mathbf{F}_{\infty} .
$$

According to the proposed process, the unique solution of SFIDE (3.1) can be denoted as

$$
\widetilde{\boldsymbol{\Psi}}_{\nu}=\mathbf{Q}_{\nu \nu}^{\nu \nu^{-1}} \mathbf{F}_{\nu}
$$


where $\boldsymbol{\Psi}_{\nu}$ is replaced by $\widetilde{\boldsymbol{\Psi}}_{\nu}$ as its approximate solution.

Subtracting (4.4) from (4.3) leads to

$$
\Psi_{\nu}-\widetilde{\Psi}_{\nu}=\mathbf{D}_{\nu \nu}^{\nu \nu} \mathbf{F}_{\nu}+\mathbf{B}_{\nu \infty}^{\nu \infty} \mathbf{F}_{\infty}
$$

where

$$
\mathbf{D}_{\nu \nu}^{\nu \nu}=\mathbf{B}_{\nu \nu}^{\nu \nu}-\mathbf{Q}_{\nu \nu}^{\nu \nu^{-1}}
$$

In the following, we expand the right-hand side of (4.5) and the first $\nu$ elements of the vector at the left-hand side of (4.5) can be expressed as

$$
\psi^{\nu}(x)-\widetilde{\psi}^{\nu}(x)=\sum_{j=0}^{m} \sum_{i=1}^{\nu} d_{i j}^{p 0}(x) f_{i}^{(j)}(x)+\sum_{j=m+1}^{\infty} \sum_{i=1}^{\nu} b_{i j}^{p 0}(x) f_{i}^{(j)}(x), \quad p=1, \ldots, \nu,
$$

where

$$
\psi^{\nu}(x)=\left[\begin{array}{c}
\psi_{1}(x) \\
\psi_{2}(x) \\
\vdots \\
\psi_{\nu}(x)
\end{array}\right], \quad \widetilde{\psi}^{\nu}(x)=\left[\begin{array}{c}
\widetilde{\psi}_{1}(x) \\
\widetilde{\psi}_{2}(x) \\
\vdots \\
\widetilde{\psi}_{\nu}(x)
\end{array}\right]
$$

and $d_{i j}^{p 0}(x), b_{i j}^{p 0}(x)$ are the elements of $\mathbf{D}_{\nu \nu}^{\nu \nu}$ and $\mathbf{B}_{\nu \infty}^{\nu \infty}$, respectively. Thus, according to the Cauchy-Schwarz inequality we have

$$
\begin{aligned}
\left|\psi^{\nu}(x)-\widetilde{\psi}^{\nu}(x)\right| \leq & \left(\sum_{j=0}^{m} \sum_{i=1}^{\nu}\left|d_{i j}^{p 0}(x)\right|^{2}\right)^{\frac{1}{2}}\left(\sum_{j=0}^{m} \sum_{i=1}^{\nu}\left|f_{i}^{(j)}(x)\right|^{2}\right)^{\frac{1}{2}} \\
& +\left(\sum_{j=m+1}^{\infty} \sum_{i=1}^{\nu}\left|b_{i j}^{p 0}(x)\right|^{2}\right)^{\frac{1}{2}}\left(\sum_{j=m+1}^{\infty} \sum_{i=1}^{\nu}\left|f_{i}^{(j)}(x)\right|^{2}\right)^{\frac{1}{2}} .
\end{aligned}
$$

It is to be noted that as $\lim _{\nu \longrightarrow \infty} \mathbf{D}_{\nu \nu}^{\nu \nu}=0$ and $\lim _{\nu \longrightarrow \infty} \mathbf{B}_{\nu \infty}^{\nu \infty}=0$, we have

$$
\lim _{\nu \longrightarrow \infty}\left|\psi^{\nu}(x)-\widetilde{\psi}^{\nu}(x)\right|=0 .
$$

\section{ILLUStRative Examples}

In this section, the efficiency and the accuracy of the proposed approach is illustrated by considering some numerical problems. The obtained numerical results are compared with some existing approaches and it was found that the proposed approximate approach produces acceptable results and even more accurate results in comparison with some existing methods. All computations are performed using Mathematica 8.

Example 5.1. Consider the following system of fractional integro-differential equations (see $[5,29])$ :

$$
\left\{\begin{array}{l}
D^{\frac{1}{2}} \psi_{1}(x)-\int_{0}^{1}\left(\psi_{1}(t)+\psi_{2}(t)\right) d t=\frac{2 \sqrt{x}}{\sqrt{\pi}}-\frac{5}{6}, \\
D^{\frac{3}{2}} \psi_{1}(x)-\int_{0}^{1}\left(\psi_{1}(t)+\psi_{2}(t)\right) d t=\frac{4 \sqrt{x}}{\sqrt{\pi}}-\frac{x}{6},
\end{array}\right.
$$


in which the initial conditions are chosen all to be zero and the exact solutions are $\psi_{1}(x)=x$ and $\psi_{2}(x)=x^{2}$.

Using the present method, the first-order and the second-order approximate solutions at equidistant points are computed. The obtained results and the results given in $[5,29]$ are listed in Tables 1 and 2. From Tables 1 and 2, we observe that the second-order approximate solution yields the exact solution as expected, since the exact solution is a polynomial function of degree 2 .

TABLE 1. Absolute errors of Example 5.1 for $\psi_{1}(x)$.

\begin{tabular}{lllll}
\hline$x$ & Method in [29] & Method in [5] & \multicolumn{1}{c}{ Suggested method } \\
\hline & & & $m=1$ & $m=2$ \\
\hline 0.1 & $8.75559 \times 10^{-2}$ & $2.78470 \times 10^{-3}$ & $1.73688 \times 10^{-1}$ & 0 \\
0.2 & $1.23823 \times 10^{-1}$ & $3.93816 \times 10^{-3}$ & $5.59324 \times 10^{-1}$ & 0 \\
0.3 & $1.51651 \times 10^{-1}$ & $4.82324 \times 10^{-3}$ & 1.98751 & 0 \\
0.4 & $1.75112 \times 10^{-1}$ & $5.56940 \times 10^{-3}$ & 4.08095 & 0 \\
0.5 & $1.95781 \times 10^{-1}$ & $6.22678 \times 10^{-3}$ & 1.10827 & 0 \\
0.6 & $2.14467 \times 10^{-1}$ & $6.82110 \times 10^{-3}$ & $5.81370 \times 10^{-1}$ & 0 \\
0.7 & $2.31651 \times 10^{-1}$ & $7.36763 \times 10^{-3}$ & $3.21226 \times 10^{-1}$ & 0 \\
0.8 & $2.47646 \times 10^{-1}$ & $7.87633 \times 10^{-3}$ & $1.50704 \times 10^{-1}$ & 0 \\
0.9 & $2.62668 \times 10^{-1}$ & $8.35411 \times 10^{-3}$ & $2.74544 \times 10^{-2}$ & 0 \\
1.0 & $2.76876 \times 10^{-1}$ & $8.80600 \times 10^{-3}$ & $6.20423 \times 10^{-2}$ & 0 \\
\hline
\end{tabular}

TABLE 2. Absolute errors of Example 5.1 for $\psi_{2}(x)$.

\begin{tabular}{lllll}
\hline $\mathrm{x}$ & Method in [5] & Method in [30] & \multicolumn{2}{c}{ Suggested method } \\
\hline & & & $m=1$ & $m=2$ \\
0.1 & $1.93140 \times 10^{-4}$ & $1.29824 \times 10^{-4}$ & $3.56504 \times 10^{-5}$ & 0 \\
0.2 & $1.09257 \times 10^{-3}$ & $3.77788 \times 10^{-4}$ & $3.25545 \times 10^{-3}$ & 0 \\
0.3 & $3.01076 \times 10^{-3}$ & $7.13496 \times 10^{-4}$ & $3.28085 \times 10^{-2}$ & 0 \\
0.4 & $6.18049 \times 10^{-3}$ & $1.12845 \times 10^{-3}$ & $1.35422 \times 10^{-1}$ & 0 \\
0.5 & $1.07969 \times 10^{-2}$ & $1.61892 \times 10^{-3}$ & $6.60271 \times 10^{-2}$ & 0 \\
0.6 & $1.70314 \times 10^{-2}$ & $2.18315 \times 10^{-3}$ & $6.09208 \times 10^{-2}$ & 0 \\
0.7 & $2.50391 \times 10^{-2}$ & $2.82043 \times 10^{-3}$ & $6.26674 \times 10^{-2}$ & 0 \\
0.8 & $3.49621 \times 10^{-2}$ & $3.53063 \times 10^{-3}$ & $6.66494 \times 10^{-2}$ & 0 \\
0.9 & $4.69331 \times 10^{-2}$ & $4.31399 \times 10^{-3}$ & $7.19976 \times 10^{-2}$ & 0 \\
1.0 & $6.10763 \times 10^{-2}$ & $5.17100 \times 10^{-3}$ & $7.88615 \times 10^{-2}$ & 0 \\
\hline
\end{tabular}

It is important to note that after converting system (5.1) into a system of linear equations, the Mathematica command 'LinearSolve' is used for the new system.

Example 5.2. Consider the following system of fractional integro-differential equations (see [29]):

$$
\left\{\begin{array}{l}
D^{\frac{1}{2}} \psi_{1}(x)-\int_{0}^{1} x \psi_{2}(t) d t=\frac{2 \sqrt{x}}{\sqrt{\pi}}-\frac{x}{2} \\
D^{\frac{1}{2}} \psi_{2}(x)-\int_{0}^{1} x \psi_{1}(t) d t=\frac{2 \sqrt{x}}{\sqrt{\pi}}-\frac{1}{3}
\end{array}\right.
$$


in which the initial conditions are chosen all to be zero and the exact solutions are $\psi_{1}(x)=x$ and $\psi_{2}(x)=x$.

We employ the approach described in Section 3 to evaluate the approximate solutions. For this case, we can find that $\psi_{m}(x)$ yields the exact solution only by setting $m=1$. Moreover, we present the results given in [29] in Table 3.

TABLE 3. Absolute errors of Example 5.2 in [29] for $\left(\psi_{1}(x), \psi_{2}(x)\right)$.

\begin{tabular}{|c|c|c|}
\hline$x$ & \multicolumn{2}{|c|}{ Methode in $[29]$} \\
\hline 0.1 & $\left(5.02704 \times 10^{-5}\right.$, & $\left.5.02704 \times 10^{-4}\right)$ \\
\hline 0.2 & $\left(1.42186 \times 10^{-4}\right.$, & $\left.7.10931 \times 10^{-4}\right)$ \\
\hline 0.3 & $\left(2.61213 \times 10^{-4}\right.$, & $\left.8.70709 \times 10^{-4}\right)$ \\
\hline 0.4 & $\left(4.02163 \times 10^{-4}\right.$, & $\left.1.00541 \times 10^{-3}\right)$ \\
\hline 0.5 & $\left(5.62040 \times 10^{-4}\right.$, & $\left.1.12408 \times 10^{-3}\right)$ \\
\hline 0.6 & $\left(7.38821 \times 10^{-4}\right.$, & $\left.1.23137 \times 10^{-3}\right)$ \\
\hline 0.7 & $\left(9.31021 \times 10^{-4}\right.$, & $\left.1.33003 \times 10^{-3}\right)$ \\
\hline 0.8 & $\left(1.13749 \times 10^{-3}\right.$, & $\left.1.42186 \times 10^{-3}\right)$ \\
\hline 0.9 & $\left(1.35730 \times 10^{-3}\right.$, & $\left.1.50811 \times 10^{-3}\right)$ \\
\hline 1.0 & $\left(1.58969 \times 10^{-3}\right.$, & $\left.1.58969 \times 10^{-3}\right)$ \\
\hline
\end{tabular}

Example 5.3. Consider the following system of fractional integro-differential equations (see $[16,30])$ :

$$
\left\{\begin{array}{l}
D^{\frac{3}{4}} \psi_{1}(x)-\int_{0}^{1}(x+t)\left[\psi_{1}(t)+\psi_{2}(t)\right] d t=-\frac{1}{20}-\frac{x}{12}+\frac{4 x^{\frac{1}{4}}}{\Gamma\left(\frac{1}{4}\right)}-\frac{128 x^{\frac{9}{4}}}{15 \Gamma\left(\frac{1}{4}\right)}, \\
D^{\frac{3}{4}} \psi_{2}(x)-\int_{0}^{1} \sqrt{x} t^{2}\left[\psi_{1}(t)-\psi_{2}(t)\right] d t=-\frac{2 \sqrt{x}}{15}-\frac{4 x^{\frac{1}{4}}}{\Gamma\left(\frac{1}{4}\right)}+\frac{32 x^{\frac{5}{4}}}{5 \Gamma\left(\frac{1}{4}\right)},
\end{array}\right.
$$

in which the initial conditions are chosen all to be zero and the exact solutions are $\psi_{1}(x)=x-x^{3}$ and $\psi_{2}(x)=x^{2}-x$.

We apply the approach described in Section 3 to determine the approximate solutions. For this case, we can find that $\psi_{m}(x)$ yields the exact solution only by setting $m=3$. We present our results when $m=1,2,3$, and the results given in [30] in Tables 4 and 5 .

Example 5.4. Consider the following system of fractional integro-differential equations (see $[16,30])$

$$
\left\{\begin{array}{l}
D^{\frac{4}{5}} \psi_{1}(x)-\int_{0}^{1} 2 x t\left[\psi_{1}(t)-\psi_{2}(t)\right] d t=\frac{83}{80} x-\frac{25 x^{\frac{6}{5}}}{3 \Gamma\left(\frac{1}{5}\right)}+\frac{125 x^{\frac{11}{5}}}{11 \Gamma\left(\frac{1}{5}\right)} \\
D^{\frac{4}{5}} \psi_{2}(x)-\int_{0}^{1}(x+t)\left[\psi_{1}(t)+\psi_{2}(t)\right] d t=-\frac{67}{160}-\frac{13}{24} x+\frac{125 x^{\frac{6}{5}}}{8 \Gamma\left(\frac{1}{5}\right)}
\end{array}\right.
$$

in which the initial conditions are chosen all to be zero and the exact solutions are $\psi_{1}(x)=x^{3}-x^{2}$ and $\psi_{2}(x)=\frac{15}{8} x^{2}$. 
TABLE 4. Absolute errors of Example 5.3 for $\psi_{1}(x)$

\begin{tabular}{lllll}
\hline$x$ & Method in [30] & & Suggested method & \\
\hline & & $m=1$ & $m=2$ & $m=3$ \\
\hline 0.1 & $1.86460 \times 10^{-3}$ & $2.33950 \times 10^{-2}$ & $4.37610 \times 10^{-3}$ & 0 \\
0.2 & $3.38103 \times 10^{-3}$ & $6.86709 \times 10^{-2}$ & $1.69027 \times 10^{-3}$ & 0 \\
0.3 & $4.91496 \times 10^{-3}$ & $1.21870 \times 10^{-1}$ & $1.70008 \times 10^{-3}$ & 0 \\
0.4 & $6.51082 \times 10^{-3}$ & $1.73108 \times 10^{-1}$ & $3.93799 \times 10^{-3}$ & 0 \\
0.5 & $8.18437 \times 10^{-3}$ & $2.11497 \times 10^{-1}$ & $4.52983 \times 10^{-3}$ & 0 \\
0.6 & $9.94249 \times 10^{-3}$ & $2.25976 \times 10^{-1}$ & $3.55933 \times 10^{-3}$ & 0 \\
0.7 & $1.17883 \times 10^{-2}$ & $2.06732 \times 10^{-1}$ & $1.36667 \times 10^{-3}$ & 0 \\
0.8 & $1.37235 \times 10^{-2}$ & $1.47035 \times 10^{-1}$ & $1.59402 \times 10^{-3}$ & 0 \\
0.9 & $1.57484 \times 10^{-2}$ & $4.52912 \times 10^{-2}$ & $4.82795 \times 10^{-3}$ & 0 \\
1.0 & $1.78631 \times 10^{-2}$ & $9.29796 \times 10^{-2}$ & $7.84433 \times 10^{-3}$ & 0 \\
\hline
\end{tabular}

TABLE 5. Absolute errors of Example 5.3 for $\psi_{2}(x)$

\begin{tabular}{lllll}
\hline$x$ & Method in [30] & & Suggested method & \\
\hline & & $m=1$ & $m=2$ & $m=3$ \\
\hline 0.1 & $1.99879 \times 10^{-4}$ & $1.46339 \times 10^{-2}$ & $3.62132 \times 10^{-3}$ & 0 \\
0.2 & $4.75397 \times 10^{-4}$ & $3.25600 \times 10^{-2}$ & $1.64100 \times 10^{-2}$ & 0 \\
0.3 & $7.89170 \times 10^{-4}$ & $4.88261 \times 10^{-2}$ & $2.95774 \times 10^{-2}$ & 0 \\
0.4 & $1.13069 \times 10^{-3}$ & $6.04406 \times 10^{-2}$ & $3.63960 \times 10^{-2}$ & 0 \\
0.5 & $1.49445 \times 10^{-3}$ & $6.45455 \times 10^{-2}$ & $3.60909 \times 10^{-2}$ & 0 \\
0.6 & $1.87697 \times 10^{-3}$ & $5.84157 \times 10^{-2}$ & $3.03835 \times 10^{-2}$ & 0 \\
0.7 & $2.27584 \times 10^{-2}$ & $3.97032 \times 10^{-2}$ & $2.15300 \times 10^{-2}$ & 0 \\
0.8 & $2.68925 \times 10^{-2}$ & $6.79901 \times 10^{-3}$ & $1.17235 \times 10^{-2}$ & 0 \\
0.9 & $3.11582 \times 10^{-2}$ & $4.07493 \times 10^{-2}$ & $2.96446 \times 10^{-3}$ & 0 \\
1.0 & $3.55442 \times 10^{-2}$ & $1.01834 \times 10^{-1}$ & $2.95048 \times 10^{-3}$ & 0 \\
\hline
\end{tabular}

Applying the approach described in this paper, we determine the approximate solutions. For this case, we can find that $\psi_{m}(x)$ yields the exact solution only by setting $m=3$. We present our numerical results obtained by proposed Taylor expansion method for $m=1,2,3$ and the results obtained in [30] in Tables 6 and 7 .

Example 5.5. Consider the following system of fractional integro-differential equations

$$
\left\{\begin{array}{l}
D^{\frac{3}{4}} \psi_{1}(x)-\int_{0}^{x} \frac{\psi_{1}(t)+\psi_{2}(t)}{\sqrt{x-t}} d t=-\frac{16 x^{\frac{5}{2}}}{15}-\frac{32 x^{\frac{7}{2}}}{35}+\frac{32 x^{\frac{5}{4}}}{5 \Gamma\left(\frac{1}{4}\right)} \\
D^{\frac{1}{2}} \psi_{2}(x)-\int_{0}^{x} \frac{\psi_{1}(t)+\psi_{2}(t)}{(x-t)^{\frac{2}{3}}} d t=-\frac{27 x^{\frac{7}{3}}}{14}+\frac{16 x^{\frac{5}{2}}}{5 \sqrt{\pi}}-\frac{243 x^{\frac{10}{3}}}{140}
\end{array}\right.
$$

in which the initial conditions are chosen all to be zero and the exact solutions are $\psi_{1}(x)=x^{2}$ and $\psi_{2}(x)=x^{3}$.

Based on the proposed method in Section 3, we obtain the approximate results by setting $m=1,2,3$ and we observe that the third-order approximate solution yields the 
TABle 6. Absolute errors of Example 5.4 for $\psi_{1}(x)$.

\begin{tabular}{lllll}
\hline$x$ & Method in [30] & & Suggested method & \\
\hline & & $m=1$ & $m=2$ & $m=3$ \\
\hline 0.1 & $1.96792 \times 10^{-4}$ & $1.66987 \times 10^{-2}$ & $4.37610 \times 10^{-3}$ & 0 \\
0.2 & $6.85268 \times 10^{-4}$ & $4.54650 \times 10^{-2}$ & $1.69027 \times 10^{-3}$ & 0 \\
0.3 & $1.42175 \times 10^{-3}$ & $7.48952 \times 10^{-2}$ & $1.70008 \times 10^{-3}$ & 0 \\
0.4 & $2.38624 \times 10^{-3}$ & $9.69101 \times 10^{-2}$ & $3.93799 \times 10^{-3}$ & 0 \\
0.5 & $3.56576 \times 10^{-3}$ & $1.05439 \times 10^{-1}$ & $4.52983 \times 10^{-3}$ & 0 \\
0.6 & $4.95084 \times 10^{-3}$ & $9.62850 \times 10^{-2}$ & $3.55933 \times 10^{-3}$ & 0 \\
0.7 & $6.53406 \times 10^{-3}$ & $6.71607 \times 10^{-2}$ & $1.36667 \times 10^{-3}$ & 0 \\
0.8 & $8.30938 \times 10^{-3}$ & $1.77783 \times 10^{-2}$ & $1.59402 \times 10^{-3}$ & 0 \\
0.9 & $1.02717 \times 10^{-2}$ & $5.00357 \times 10^{-2}$ & $4.82795 \times 10^{-3}$ & 0 \\
1.0 & $1.24167 \times 10^{-2}$ & $1.32209 \times 10^{-1}$ & $7.84433 \times 10^{-3}$ & 0 \\
\hline
\end{tabular}

TABLE 7. Absolute errors of Example 5.4 for $\psi_{2}(x)$.

\begin{tabular}{lllll}
\hline$x$ & Method in [30] & & Suggested method & \\
\hline & & $m=1$ & $m=2$ & $m=3$ \\
\hline 0.1 & $8.20450 \times 10^{-4}$ & $1.35222 \times 10^{-1}$ & $4.98795 \times 10^{-2}$ & 0 \\
0.2 & $1.58553 \times 10^{-3}$ & $1.88478 \times 10^{-1}$ & $8.22827 \times 10^{-2}$ & 0 \\
0.3 & $2.41026 \times 10^{-3}$ & $2.17328 \times 10^{-1}$ & $9.64328 \times 10^{-2}$ & 0 \\
0.4 & $3.30743 \times 10^{-3}$ & $2.25836 \times 10^{-1}$ & $9.56954 \times 10^{-2}$ & 0 \\
0.5 & $4.28071 \times 10^{-3}$ & $2.16061 \times 10^{-1}$ & $8.41589 \times 10^{-2}$ & 0 \\
0.6 & $5.33111 \times 10^{-3}$ & $1.89798 \times 10^{-1}$ & $6.57542 \times 10^{-2}$ & 0 \\
0.7 & $6.45864 \times 10^{-3}$ & $1.49181 \times 10^{-1}$ & $4.42508 \times 10^{-2}$ & 0 \\
0.8 & $7.66286 \times 10^{-3}$ & $9.71051 \times 10^{-2}$ & $2.32810 \times 10^{-2}$ & 0 \\
0.9 & $8.94313 \times 10^{-3}$ & $3.76493 \times 10^{-2}$ & $6.32948 \times 10^{-3}$ & 0 \\
1.0 & $1.02987 \times 10^{-2}$ & $2.34213 \times 10^{-2}$ & $3.30327 \times 10^{-3}$ & 0 \\
\hline
\end{tabular}

exact solution as expected. In the following, our results for $m=1,2,3$ at equidistant points in $[0,1]$ are tabulated in Tables 8 and 9.

TABle 8. Absolute errors of Example 5.5 for $\psi_{1}(x)$.

\begin{tabular}{llll}
\hline$x$ & $m=1$ & $m=2$ & $m=3$ \\
\hline 0.1 & $4.39572 \times 10^{-4}$ & $5.63735 \times 10^{-8}$ & 0 \\
0.2 & $2.02649 \times 10^{-3}$ & $1.49505 \times 10^{-6}$ & 0 \\
0.3 & $6.38129 \times 10^{-3}$ & $1.61418 \times 10^{-5}$ & 0 \\
0.4 & $1.85611 \times 10^{-2}$ & $1.16368 \times 10^{-4}$ & 0 \\
0.5 & $4.69815 \times 10^{-2}$ & $6.32737 \times 10^{-4}$ & 0 \\
0.6 & $9.46103 \times 10^{-2}$ & $2.86770 \times 10^{-3}$ & 0 \\
0.7 & $1.53109 \times 10^{-1}$ & $1.22967 \times 10^{-2}$ & 0 \\
0.8 & $2.14122 \times 10^{-1}$ & $7.00457 \times 10^{-2}$ & 0 \\
0.9 & $2.76101 \times 10^{-1}$ & $2.65058 \times 10^{-1}$ & 0 \\
1.0 & $3.40830 \times 10^{-1}$ & $1.19614 \times 10^{-1}$ & 0 \\
\hline
\end{tabular}


TABle 9. Absolute errors of Example 5.5 for $\psi_{2}(x)$.

\begin{tabular}{llll}
\hline$x$ & $m=1$ & $m=2$ & $m=3$ \\
\hline 0.1 & $1.17689 \times 10^{-4}$ & $2.02948 \times 10^{-5}$ & 0 \\
0.2 & $1.61962 \times 10^{-3}$ & $1.53357 \times 10^{-4}$ & 0 \\
0.3 & $9.65962 \times 10^{-3}$ & $4.69785 \times 10^{-4}$ & 0 \\
0.4 & $3.89089 \times 10^{-2}$ & $8.58738 \times 10^{-4}$ & 0 \\
0.5 & $1.13095 \times 10^{-1}$ & $4.68815 \times 10^{-4}$ & 0 \\
0.6 & $2.40454 \times 10^{-1}$ & $4.31091 \times 10^{-3}$ & 0 \\
0.7 & $3.98040 \times 10^{-1}$ & $2.88928 \times 10^{-2}$ & 0 \\
0.8 & $5.63382 \times 10^{-1}$ & $1.89377 \times 10^{-1}$ & 0 \\
0.9 & $7.33038 \times 10^{-1}$ & $7.60116 \times 10^{-1}$ & 0 \\
1.0 & $9.12716 \times 10^{-1}$ & $3.53191 \times 10^{-1}$ & 0 \\
\hline
\end{tabular}

\section{Conclusion}

In this paper, we have proposed an approximate method for solving systems of fractional integro-differential equations. In the proposed technique, the SFIDE to be solved, has been converted into integral equations. Then Taylor expansion for unknown functions and integration method have employed to convert the resulting integral equations into a system of linear equations with respect to unknown functions and their derivatives. By applying a standard method the resulting system has been solved. In particular for such cases when the exact solutions are polynomial functions of degree up to $m$, the derived $m$ th-order approximations are exact.

\section{REFERENCES}

[1] R. B. Albadarneh, M. Zerqat and I. M. Batiha, Numerical solutions for linear and non-linear fractional differential equations, International Journal of Pure and Applied Mathematics 106(3) (2016), 859-871.

[2] Q. M. Al-Mdallal, M. I. Syam and M. N. Anwar, A collocation-shooting method for solving fractional boundary value problems, Commun. Nonlinear. Sci. Numer. Simul. 15 (2010), 38143822 .

[3] F. Ghoreishi and P. Mokhtary, Spectral collocation method for multi-order fractional differential equations, Int. J. Comput. Methods 11(5) (2014), Paper ID 1350072, 23 pages.

[4] A. Golbabai and K. Sayevand, Analytical treatment of differential equations with fractional coordinate derivatives, Comput. Math. Appl. 62 (2011), 1003-1012.

[5] S. A. Deif and S. R. Grace, Iterative refinement for a system of linear integro-differential equations of fractional type, J. Comput. Appl. Math, DOI 10.1016/j.cam.2015.08.008.

[6] M. Didgar and N. Ahmadi, An efficient method for solving systems of linear ordinary and fractional differential equations, Bull. Malays. Math. Sci. Soc. 38(4) (2015), 1723-1740.

[7] K. Diethelm, The Analysis of Fractional Differential Equations, Springer-Verlag, Berlin, Heidelberg, New York, 2010.

[8] Y. Huang and X.-F. Li, Approximate solution of a class of linear integro-differential equations by Taylor expansion method, Int. J. Comput. Math. 87(6) (2010), 1277-1288.

[9] L. Huang, X.-F. Li and Y. Huang, Approximate solution of Abel integral equation, Comput. Math. Appl. 56 (2008), 1748-1757.

[10] L. Huang, X.-F. Li, Y. Zhao and X.-Y. Duan, Approximate solution of fractional integrodifferential equations by Taylor expansion method, Comput. Math. Appl. 62 (2011), 1127-1134. 
[11] M. Inc, The approximate and exact solutions of the space-and time-fractional Burgers equations with initial conditions by variational iteration method, J. Math. Anal. Appl. 345 (2008), 476-484.

[12] H. Jafari, A. Golbabai, S. Seifi and K. Sayevand, Homotopy analysis method for solving multiterm linear and nonlinear diffusion-wave equations of fractional order, Comput. Math. Appl. 59 (2010), 1337-1344.

[13] A. A. Kilbas, H. M. Srivastava and J. J. Trujillo, Theory and Applications of Fractional Differential Equations, North-Holland, Mathematics Studies 204, Elsevier Science B.V. Amsterdam, 2006.

[14] X.-F. Li, Approximate solution of linear ordinary differential equations with variable coefficients, Math. Comput. Simulation 75 (2007), 113-125.

[15] X.-F. Li, L. Huang and Y. Huang, A new Abel inversion by means of the integrals of an input function with noise, J. Phys. A 40 (2007), 347-360.

[16] A. M. S. Mahdy and E. M. H. Mohamed, Numerical studies for solving system of linear fractional integro-differential equations by using least squares method and shifted Chebyshev polynomials, Journal of Abstract and Computational Mathematics 1 (2015), 24-32.

[17] K. Maleknejad and T. Damercheli, Improving the accuracy of solutions of the linear second kind volterra integral equations system by using the Taylor expansion method, Indian J. Pure Appl. Math. 45(3) (2014), 363-376.

[18] K. S. Miller and B. Ross, An Introduction to the Fractional Calculus and Fractional Differential Equations, Wiley-Interscience Publication, John Wiley and Sons, New York, Chichester, Brisbane, Toronto, 1993.

[19] S. Momani, Analytic and approximate solutions of the space- and time-fractional telegraph equations, Appl. Math. Comput. 170 (2005), 1126-1134.

[20] S. Momani and Z. Odibat, Analytical approach to linear fractional partial differential equations arising in fluid mechanics, Phys. Lett. A 355 (2006), 271-279.

[21] S. Momani and Z. Odibat, Analytical solution of a time-fractional Navier-Stokes equation by Adomian decomposition method, Appl. Math. Comput. 177 (2006), 488-494.

[22] S. Momani and Z. Odibat, Comparison between the homotopy perturbation method and the variational iteration method for linear fractional partial differential equations, Comput. Math. Appl. 54 (2007), 910-919.

[23] Z. Odibat and S. Momani, An algorithm for the numerical solution of differential equations of fractional order, J. Appl. Math. Inform. 26 (2008), 15-27.

[24] K. B. Oldham and J. Spanier, The Fractional Calculus, Academic Press, New York, 1974.

[25] K. Parand and M. Nikarya, Application of Bessel functions for solving differential and integrodifferential equations of the fractional order, Appl. Math. Model. 38 (2014), 4137-4147.

[26] I. Podlubny, Fractional Differential Equations, Academic Press, New York, 1999.

[27] E. A. Rawashdeh, Numerical solution of semidifferential equations by collocation method, Appl. Math. Comput. 174 (2006), 869-876.

[28] M. Rehman and R. A. Khan, Numerical solutions to initial and boundary value problems for linear fractional partial differential equations, Appl. Math. Model. 37 (2013), 5233-5244.

[29] R. K. Saeed and H. M. Sdeq, Solving a system of linear Fredholm fractional integro-differential equations using homotopy perturbation method, Australian Journal of Basic and Applied Sciences 4 (2010), 633-638.

[30] M. H. Saleh, S. H. Mohamed, M. H. Ahmed and M. K. Marjan, System of linear fractional integro-differential equations by using Adomian decomposition method, Int. J. Comput. Appl. 121(24) (2015), 9-19.

[31] B.-Q. Tang and X.-F. Li, A new method for determining the solution of Riccati differential equations, Appl. Math. Comput. 194 (2007), 431-440.

[32] B.-Q. Tang and X.-F. Li, Approximate solution to an integral equation with fixed singularity for a cruciform crack, Appl. Math. Lett. 21 (2008), 1238-1244. 
[33] A. R. Vahidi and M. Didgar, An improved method for determining the solution of Riccati equations, Neural Comput. 23 (2013), 1229-1237.

[34] W. K. Zahra and S. M. Elkholy, The use of cubic splines in the numerical solution of fractional differential equations, Int. J. Math. Math. Sci. 16 (2012), DOI 10.1155/2012/638026.

${ }^{1}$ Department of Mathematics,

Guilan Science and Research Branch,

Islamic AzAD University, RASHT, IrAN

${ }^{2}$ Department of Mathematics,

RASHT BRANCH,

ISLAMIC AZAD UNIVERSITY, RASHT, IRAN

Email address: mohsen_didgar@yahoo.com

${ }^{3}$ Department of Mathematics,

College of Science, Yadegar-e-Emam Khomeyni (RAH) Shahr-e-Rey Branch, Islamic AzAd University, TEHRAN, IRAN

Email address: alrevahidi@yahoo.com

${ }^{4}$ Department of Applied Mathematics,

FACUlty of Mathematical Sciences,

University of Guilan, P.O. Box 41335-1914, P.C.4193822697, Rasht, Iran

Email address: j.biazar@gmail.com

*Corresponding Author 